\title{
Acute Liver Failure Due To Amoxicillin and Amoxicillin/Clavulanate
}

\author{
ROBERT J. FONTANA, MD,* A. OBAID SHAKIL, MD,† JOEL K. GREENSON, MD, \\ and WILLIAM M. LEE, MD
}

\begin{abstract}
The aim of our study is to report upon the presentation of two patients with life-threatening acute liver failure (ALF) due to amoxicillin and amoxicillin/clavulanate. A 59-year-old, Caucasian male presented with ALF 34 days after receiving amoxicillin/clavulanate. Despite aggressive supportive care, he died on hospital day 10. A 42-year-old, Caucasian female presented with ALF 21 days after receiving amoxicillin. She underwent successful liver transplantation on hospital day 19. In both cases, all competing causes of ALF had been excluded, liver pathology was consistent with druginduced hepatitis, and cases were deemed "definite/highly probable" using causality assessment. Amongst 14 prior ALF/death cases due to amoxicillin/clavulanate, the mean age (62 years), male predominance $(57 \%)$, and mean delay from drug cessation to presentation (17 days) is similar to what has been reported in patients with self-limited cholestatic hepatitis. Acute liver failure is a rare manifestation of amoxicillin and amoxicillin/clavulanate hepatotoxicity with no obvious clinical features at presentation portending a poor prognosis. Early transfer of patients with severe druginduced hepatotoxicity (i.e., encephalopathy or coagulopathy) to a transplant center is recommended due to their poor likelihood of recovery.
\end{abstract}

KEY WORDS: amoxicillin/clavulanate; acute liver failure; transplantation; hepatotoxicity.

Ampicillin and amoxicillin are commonly used semisynthetic penicillins that are associated with a very low rate of mild hepatocellular and cholestatic liver injury when used alone $(1,2)$. However, when amoxicillin is combined with the B-lactamase inhibitor, clavulanic acid or clavulanate (Augmentin ${ }^{\mathrm{TM}}$, GlaxoSmithKline, Research Triangle Park, NC), the estimated risk of hepatotoxicity increases from 3 to 17 per 100,000 prescriptions presumably due to the clavulanate component (2). Men over the age of 50 appear to be at increased risk of amoxicillin/clavulanate hepatotoxicity as are patients who receive prolonged or re-

\footnotetext{
Manuscript received November 12, 2004; accepted January 18, 2005. From the *Department of Internal Medicine, $\ddagger$ Department of Pathology, University of Michigan Medical Center, Ann Arbor, Michigan, USA; and †Department of Medicine, University of Pittsburgh, Pittsburgh, Pennsylvania, §Adverse Drug Reactions Unit, Therapeutic Goods Administration, Canberra, Australia; and TDepartment of Medicine, University of Texas Southwestern, Dallas, Texas, USA.

Address for reprint requests: Robert J. Fontana, MD, 3912 Taubman Center, Ann Arbor, Michigan 48109-0362, USA; rfontana@umich.edu.
}

peated courses of treatment (2). A delayed cholestatic or mixed hepatocellular-cholestatic liver injury pattern has typically been reported with amoxicillin/clavulanate that usually has a benign course and resolves within 2 months (2-5).

Acute liver failure (ALF) is a rare but devastating illness defined as the onset of coagulopathy and encephalopathy within 8 weeks of presentation in a patient without known underlying liver disease (6). Although a multitude of causes of ALF have been identified, a prospective study of 309 consecutive ALF patients demonstrated that acetaminophen (33\%) and idiosyncratic drug-induced hepatotoxicity $(13 \%)$ were the most commonly identified causes of ALF in the United States (7). Although most patients with amoxicillin and amoxicillin/clavulanate hepatotoxicity recover with supportive care, we recently observed two patients with life-threatening ALF because of these medications. The aim of this study is to report on the presentation and clinical course of two patients with ALF due to amoxicillin and amoxicillin/clavulanate. We 
also set out to identify potential features associated with severe outcomes by reviewing the published literature.

\section{METHODS}

The United States Acute Liver Failure Study Group (ALFSG) is a consortium of 24 referral centers studying the etiologies, natural history, and outcomes of patients with ALF. Between January 1998 and August 2003, 610 consecutive ALF patients were enrolled into a 3-week observational study including 76 patients with idiosyncratic drug-induced hepatotoxicity. Etiological diagnoses were made at each study center using accepted diagnostic criteria (7). Laboratory, clinical, and outcome data were collected from all participants at death, liver transplantation, or 3 weeks of follow-up. The research protocol was reviewed and approved by the Institutional Review Board of the participating institutions and written informed consent was obtained from the next of kin of all participants. A Certificate of Confidentiality was obtained for the entire study.

\section{Causality Assessment}

To standardize the diagnostic criteria for drug-induced liver injury, several scoring systems have been proposed $(8,9)$. The Council for International Organization of Medical Sciences (CIOMS) scale uses seven clinical criteria to generate a score that varies between -5 and +14 and is categorized into five levels of causality : $>8$ definite/highly probable, $6-8$ probable, $3-5$ possible, $1-2$ unlikely, and $\leq 0$ excluded. The CIOMS score has been validated by applying data from a large number of patients who had been rechallenged with the suspect agent $(10,11)$. The Clinical Diagnostic Scale (CDS) provides a score between 6 and 20 that can be categorized into 4 levels of causality: $>17$ definite, 14-16 probable, 10-13 possible, and 6-9 unlikely. The CDS was validated using cohorts of real cases, fictitious cases, and the opinions of an external expert panel $(12,13)$.

\section{RESULTS}

\section{Case \#1}

A 59-year old, Caucasian male presented to his primary care physician with cough and fatigue in early June '03. The patient had a history of hypertension treated with atenolol $100 \mathrm{mg}$ per day and triamterene/hydrochlorothiazide $75 / 50 \mathrm{mg}$ per day for the past year. Hyperlipidemia was treated with atorvastatin $20 \mathrm{mg}$ per day for 5 years and the patient also received monthly testosterone injections ( $400 \mathrm{mg}$ for 5 years) and sildenafil $25 \mathrm{mg}$ as needed for impotence. The patient reported taking hydrocodone/acetaminophen $5 / 500 \mathrm{mg}$ and naproxen $500 \mathrm{mg}$ once a day as needed for osteoarthritis of the knee. He had no known drug allergies. On 6/6/03, amoxicillin/clavulanate $500 / 125 \mathrm{mg}$ twice a day was prescribed for scalp cellulitis which he took for 12 days. On 6/25/03, routine labs revealed an AST 107 IU/L, ALT $141 \mathrm{IU} / \mathrm{L}$, alkaline phosphatase $98 \mathrm{IU} / \mathrm{L}$, INR 1.0 and normal total bilirubin. In evaluating his abnormal serum aminotransferase levels, hepatitis A, B, C, epstein-barr virus, cytomegalovirus, anti-nuclear (ANA) and smooth muscle antibodies were all negative or undetectable. In addition, serum ceruloplasmin, alpha-1 anti-trypsin, and iron levels were normal as was a liver ultrasound. Because of persistent scalp erythema, he was prescribed amoxicillin alone $500 \mathrm{mg}$ three times a day on 7/5/03 which he took for 3 days.

On 7/22/03 he was hospitalized with generalized malaise, vomiting, and jaundice. On physical examination, there were was a scaly rash involving the scalp and legs but no evidence of ascites or stigmata of chronic liver disease. The patient denied a history of recent alcohol ingestion, illicit drug use, travel, or consumption of over the counter or herbal products. His admission serum ALT was $620 \mathrm{IU} / \mathrm{L}$, AST $628 \mathrm{IU} / \mathrm{L}$, alkaline phosphatase $170 \mathrm{IU} / \mathrm{L}$ and bilirubin $27.6 \mathrm{mg} / \mathrm{dL}$ with INR 2.0. His admission WBC was 12,600 cells $/ \mathrm{mL}$ with no eosinophils. The ANA, smooth muscle antibody, and anti-mitochondrial antibodies were negative and HCV RNA and HBV DNA were undetectable. Over the next 3 days, the patient developed worsening hepatic encephalopathy and coagulopathy consistent with ALF. A transjugular liver biopsy on hospital day \# 6 revealed acute hepatitis with confluent necrosis predominating in the central zones consistent with a drug reaction (Figure 1). Because of his worsening liver function, he was placed on the liver transplant waiting list on hospital day \#8. Despite antibiotics and supportive care, he developed respiratory failure and refractory hypotension necessitating intubation, pressors, and continuous venovenous hemofiltration and he died 2 days later of multiorgan failure. Permission for an autopsy was denied.

\section{Case \#2}

A 42-year old Caucasian female presented on 2/20/01 with a 4-week history of malaise and jaundice. The patient had received hydrocodone/ibuprofen $7.5 / 200 \mathrm{mg}$ per day and amoxicillin $500 \mathrm{mg}$ three times a day for 6 days in late January 2001 following dental work for an infected tooth. At admission to the outside hospital on 2/20/01, serum AST was $1189 \mathrm{IU} / \mathrm{mL}$, ALT $521 \mathrm{IU} / \mathrm{mL}$, gammaglutamyl transpeptidase (GGTP) $89 \mathrm{IU} / \mathrm{mL}$, and bilirubin $23.0 \mathrm{mg} / \mathrm{dL}$ with INR of 1.4 The WBC was 6000 cells $/ \mathrm{mL}$ with no eosinophilia. The patient was deeply jaundiced but there was no rash, adenopathy, ascites, or stigmata of chronic liver disease. She denied a history of drug allergies, alcohol use, illicit drug use, recent travel, or prior medical history. Laboratory evaluation included negative serologies for hepatitis A, B, C, ANA, and anti-smooth muscle antibody. Serum ceruloplasmin was normal. She was subsequently transferred 

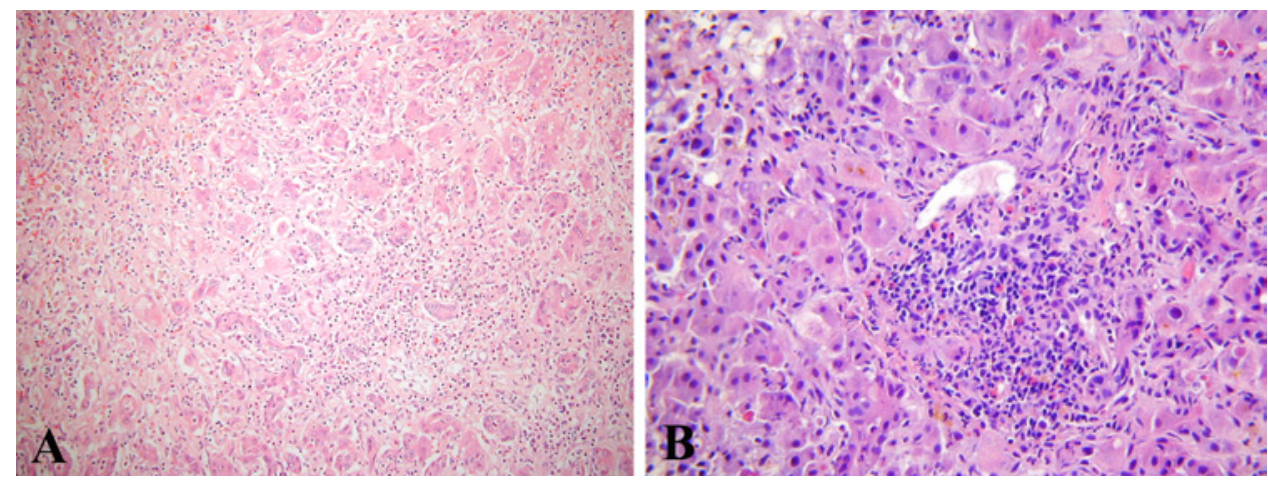

Fig 1. Liver histology from patient \#1 (A) and patient \#2 (B). A). This low power photomicrograph shows central zone necrosis (upper left) with neocholangiogenesis and mild portal inflammation B). This high-power photomicrograph shows portal infiltrates of lymphocytes and eosinophils. Adjacent hepatocytes show ballooning degeneration and cholestasis.

to the University of Pittsburgh Medical Center for further evaluation on $2 / 22 / 01$. An ultrasound guided liver biopsy on hospital day \#4 revealed expansion of the portal tracts with a lymphoplasmacytic infiltrate, eosinophils and mild ductular proliferation suggestive of a drug reaction. Over the ensuing 10 days, she developed progressive hepatic encephalopathy requiring intubation and was listed for a liver transplant on hospital day \#18 for druginduced ALF. At listing, her serum ALT was $766 \mathrm{IU} / \mathrm{L}$, AST $2075 \mathrm{IU} / \mathrm{L}$, alkaline phosphatase $135 \mathrm{IU} / \mathrm{mL}$, and bilirubin $26.9 \mathrm{mg} / \mathrm{dL}$ with INR 2.0. An orthotopic liver transplant was performed on hospital day \#19. The patient was discharged home on hospital day \# 38 in stable condition and is alive and well as of 9/04. Her explanted liver weighed $1650 \mathrm{~g}$ and showed submassive hepatic necrosis. There were numerous areas of confluent central zone necrosis with collapse and neocholangiogenesis consistent with a drug reaction (Figure 1).

\section{Causality Assessment}

Patient \#1 had a CIOMS score of 9 which is "definite/highly probable" and a CDS score of 13 which is "possible" (Table 1). Similarly, patient \#2 had a CIOMS score of 9 which is "definite/highly probable" and a CDS score of 9 which is "unlikely."

\section{DISCUSSION}

We present two patients who developed life threatening ALF associated with a short course of amoxicillin/clavulanate and amoxicillin, respectively. The dose and duration of antibiotics were within the labeling indications and neither of the patients had a history of known allergy or intolerance to B-lactam antibiotics, alcohol use, illicit drug use, or prior liver disease. A thorough evaluation for competing causes of liver injury was negative in both instances. The development of hepatitis 4-6 weeks after drug ingestion in both cases is consistent with prior reports of B-lactam associated hepatotoxicity $(2,3)$. Neither patient had prominent features of a hypersensitivity reaction such as fever and eosinophilia that has been reported in up to $50 \%$ of patients with amoxicillin/clavulanate hepatotoxicity $(4,5)$. However, review of available liver histology demonstrated histological features compatible with a severe drug induced hepatitis in both cases (Figure 1).

Establishing a diagnosis of drug induced liver injury requires a careful exclusion of competing causes and an awareness of the hepatotoxicity profile of the suspect agent. Both amoxicillin and amoxicillin/clavulanate are widely prescribed antibiotics with an estimated 34 million and 15 million prescriptions dispensed in the United States alone in 2002, respectively (14). There have been multiple

TABle 1. CiOMS and CDS Scores of Case Patients

\begin{tabular}{lrr}
\hline & Case 1 & Case 2 \\
\hline ClOMS & & \\
1. Time to onset & +3 & +3 \\
2. Course of reaction & +2 & +2 \\
3. Risk factor for drug reaction & +1 & 0 \\
4. Concomitant drugs & -1 & 0 \\
5. Nondrug-related causes & +2 & +2 \\
6. Previous information on drug & +2 & +2 \\
7. Response to readministration & 0 & 0 \\
Total Score & +9 & +9 \\
Clinical Diagnostic Scale (CDS) & & \\
1. Temporal relationship & & \\
a. Time to onset & +3 & +3 \\
b. Time from withdrawal & +3 & 0 \\
c. Time to normalize & 0 & 0 \\
2. Nondrug-related causes & +3 & +3 \\
3. Extrahepatic manifestations & +2 & +1 \\
4. Response to readministration & 0 & 0 \\
5. Previous information on drug & +2 & +2 \\
Total Score & +13 & +9 \\
\hline
\end{tabular}


published reports of amoxicillin/clavulanate associated cholesatic hepatitis since its introduction in the early 1980s (2-6). A recent literature review indicates a male predominance $(70 \%)$ with a mean age of onset of 60 years (range: 1-90) (15). The mean delay between drug exposure and presentation is 4 weeks with a range of 18 weeks which can lead to a delay in diagnosis. The clinical presentation of patient \#1 is consistent with amoxicillin/clavulanate hepatotoxicity since all other known causes of ALF had been excluded and the patient was an elderly male who received a prolonged course of antibiotic treatment. Although the patient was receiving other potentially hepatotoxic medications (i.e., testosterone, atorvastatin, naproxen), these medications had been used for prolonged periods of time and the temporal relationship between amoxicillin/clavulanate use was most compatible with a drug reaction. As previously mentioned, amoxicillin alone is rarely identified as a cause of liver injury yet alone ALF making causality assessment in case \#2 more challenging (1). However, this individual was previously healthy and the absence of other identifiable etiologies including other hepatotoxic medications and the timing of her presentation after taking amoxicillin with liver histology highly suggestive of an acute drug induced hepatitis strongly support a diagnosis of amoxicillin-induced ALF.

To improve diagnostic accuracy, causality assessment instruments have been proposed for patients with suspected drug-induced liver injury $(8,9)$. Since there is no "gold standard" laboratory or diagnostic test, these instruments are usually validated by comparing results to that of an experienced expert panel. In this report, formal causality assessment supported a diagnosis of drug induced liver injury in both cases using the CIOMS instrument (Table 1). Although case \#2 was scored as "unlikely" using the CDS scale, it has previously been shown that the CDS scale is most useful in patients with an acute immunoallergic illness and less helpful in patients with metabolic idiosyncrasy or a delayed presentation (13). In addition, the CDS scale is not useful in assessing causality in patients with ALF or fatal hepatotoxicity as was seen in these two patients (13). These cases demonstrate the limitations of the currently available causality assessment instruments and the need to develop more accurate and sensitive instruments.

The natural history of amoxicillin/clavulanate hepatotoxicity has been characterized as generally mild and nearly always self-limited $(3,4)$. A systematic review of 208 cases demonstrated that normalization of liver biochemistries occurred after a mean of 11.5 weeks (14). Rare instances of chronic liver injury with ductopenia and prolonged cholestasis have been reported $(3,4)$. With an estimated incidence of 17 per 100,000 pre- scriptions and 14 million Americans receiving amoxicillin/clavulanate each year, one may anticipate up to 2400 cases of cholestatic hepatitis associated with this agent each year. The two patients in the present report represented $0.3 \%$ ( 2 of 610 ) of the ALF cases observed during the study period by the US ALFSG. Since the centers participating in the US ALFSG account for $~ 30 \%$ of the liver transplant activity and capture 100 of the 2000 annual ALF cases in the United States, one would expect a maximum of six ALF cases due to amoxicillin and amoxicillin/clavulanate in the United States on an annual basis. These estimates combined with the large number of patients taking these drugs on a daily basis suggest that life-threatening hepatotoxicity due to amoxicillin and amoxicillin/clavulanate is indeed rare.

A MEDLINE search using the keywords amoxicillin and amoxicillin/clavulanate crossed with liver transplantation, acute liver failure, toxic hepatitis, and death identified only six published articles describing 14 fatalities in patients receiving amoxicillin/clavulanate and none due to amoxicillin alone (16-22) (Table 2). Several of the patients had prominent extrahepatic manifestations such as Steven's Johnson Syndrome, interstitial nephritis, and thrombotic thrombocytopenic purpura emphasizing the complex, systemic nature of some of these severe drug reactions $(16,18,22)$. In 1996, the Australian Adverse Drug Reactions Advisory Committee (ADRAC) reported nine Australian patients with amoxicillin/clavulanate hepatotoxicity that had died (21). However, in two of the cases the cause of death was not felt to be drug-related and in two others the primary cause of death was not liver related while the remaining five died of liver failure. As of October 2003, the ADARC had recorded 18 fatal cases of amoxicillin/clavulanate in its registry (Ian Boyd personal communication, February 2004). Review of the published cases along with ours demonstrates that there is a male predominance (57\%) and the mean subject age is 62 years (range: 27-91) amongst ALF/fatal cases. In addition, the mean time from completing amoxicillin/clavulante to presentation was 17 days (range: 0-34) which is similar to that of patients with self-limited amoxicillin/clavulanate cholestatic hepatitis. Therefore, there does not appear to be any obvious clinical risk factors for severe outcomes versus self-limited hepatotoxicity from amoxicillin/clavulanate and one may anticipate that ALF will continue to be an exceedingly rare and unpredictable manifestation of amoxicillin/clavulanate hepatotoxicity.

It is estimated that $\sim 10 \%$ of patients who develop jaundice due to severe drug induced liver injury will die and that elderly patients are more susceptible to drug induced liver injury as well as poor outcomes $(23,24)$. Recent studies also suggest that patients with ALF due to idiosyncratic 
TABle 2. ACute Liver Failure And Mortality RePorted with Amoxicillin/Clavulanate

\begin{tabular}{|c|c|c|c|c|c|}
\hline Series (ref\#) & Indication (days) & $\operatorname{Delay}(d)^{*}$ & Age/sex & Ethnicity & Outcome \\
\hline \multirow[t]{7}{*}{ Australia $(19,21)$} & Respiratory (28) & 21 & $37 \mathrm{~F}$ & NA & $\begin{array}{l}\text { Death-Liver related. Also received } \\
\text { valproate. }\end{array}$ \\
\hline & Bronchitis (2) & 0 & $61 \mathrm{~F}$ & NA & Death-Liver related \\
\hline & NA & NA & NA F & NA & Death-Liver related \\
\hline & Urinary (10) & 33 & $69 \mathrm{~F}$ & NA & Death-Liver related \\
\hline & Respiratory (10) & 3 & $60 \mathrm{M}$ & NA & $\begin{array}{l}\text { Death-Heart failure and pulmonary } \\
\text { embolism }\end{array}$ \\
\hline & Respiratory(NA) & NA & $88 \mathrm{M}$ & NA & $\begin{array}{l}\text { Death-Pneumonia with severe } \\
\text { cholestasis }\end{array}$ \\
\hline & Urinary (28) & 7 & $81 \mathrm{M}$ & $\mathrm{C}$ & $\begin{array}{l}\text { Death-Received ethinyl estradiol } \\
\text { and biopsy had cholestasis, died in } \\
9 \text { weeks }\end{array}$ \\
\hline \multirow[t]{2}{*}{ Turkey (18) } & Bronchitis (5) & 1 & $40 \mathrm{~F}$ & $\mathrm{C}$ & Survived-Discharged on day 23 \\
\hline & Pharyngitis (5) & 1 & $66 \mathrm{M}$ & $\mathrm{C}$ & $\begin{array}{l}\text { Death-Thrombotic } \\
\text { thrombocytopenic purpura \& } \\
\text { hemolytic anemia refractory to } \\
\text { steroids }\end{array}$ \\
\hline United States (16) & Pneumonia (10) & 32 & $38 \mathrm{M}$ & $\mathrm{C}$ & $\begin{array}{l}\text { Death-Interstitial nephritis and } \\
\text { Stevens-Johnson refractory to } \\
\text { steroids, died in } 10 \text { weeks }\end{array}$ \\
\hline United States (22) & Pneumonia (4) & 28 & $37 \mathrm{M}$ & $\mathrm{C}$ & $\begin{array}{l}\text { Death-Toxic epidermal necrolysis } \\
\text { present, died of multiorgan failure } \\
\text { at } 3 \text { months }\end{array}$ \\
\hline Scotland (20) & NA & NA & $91 \mathrm{~F}$ & $\mathrm{C}$ & $\begin{array}{l}\text { Death-Recent hip surgery with } \\
\text { resolving cholestasis }\end{array}$ \\
\hline Spain (17) & Dental (7) & 28 & $80 \mathrm{M}$ & $\mathrm{C}$ & $\begin{array}{l}\text { Survived-Steroids given, recovered } \\
\text { within } 2 \text { months }\end{array}$ \\
\hline United States* & Cellulitis (12) & 34 & $59 \mathrm{M}$ & $\mathrm{C}$ & Death-Liver related, died on day 10 \\
\hline
\end{tabular}

Note. $\mathrm{C}=$ Caucasian $\mathrm{NA}=$ Not available.

$*$ Current report Delay $=$ Days from completing amoxicillin/clavulanate to presentation.

drug reactions have a poor prognosis as a group independent of the suspect agent or subject age with only a $20 \%$ likelihood of spontaneous survival (7). Therefore, when clinicians encounter a patient with severe drug induced liver injury that meets criteria for ALF, they should have a low threshold for transferring the patient to a transplant center for evaluation early in their course $(6,7)$.

The mechanism of amoxicillin and amoxicillin/clavulanate hepatotoxicity remains uncertain. The clavulinic acid component is suspected of being responsible for the liver injury since rechallenge with amoxicillin alone is well tolerated while rechallenge with amoxicillin/clavulanate is not (2). In addition, clavulanate combined with other B-lactams can also lead to liver injury (25). The ratio of amoxicillin to clavulanate varies from $8: 1$ to $2: 1$ in different formulations but the importance of the ratio in determining the frequency and severity of hepatotoxicity is unknown $(26,27)$. Recently, a genetic basis for amoxicillin/ clavulante hepatotoxicity has been identified with linkage to the human leukocyte antigen (HLA) haplotype particularly in patients with immunoallergic and cholestatic features $(20,28)$. In patient \#2, the serological HLA haplotype was not similar to those reported to predispose to hepatotoxicity (Data not shown). Unfortunately, HLA data was not available from patient \#1.

In summary, we present two patients with severe hepatotoxicity due to amoxicillin/clavulanate and amoxicillin that rapidly progressed to life-threatening ALF. Although the majority of patients with amoxicillin/clavulanate hepatotoxicity have an acute self-limited illness, we recommend that all patients with suspected drug induced ALF be referred to a transplant center for urgent evaluation due to their low rate of spontaneous survival (7). To facilitate further research on the clinical, environmental, genetic and immunological basis of idiosyncratic drug-induced hepatotoxicity, prospective studies that collect biological samples from well-characterized patients compared to unaffected controls is recommended.

\section{ACKNOWLEDGMENTS}

The authors would like to acknowledge members of the US Acute Liver Failure Study Group 1998-2003. William M. Lee (PI), Frank V. Schiødt, George Ostapowicz, University of Texas Southwestern Medical Center, Dallas, TX; Anne M. Larson, University of Washington, Seattle, WA; Raj Santyanarayana, Cary Caldwell, Lawton Shick, Washington University, St Louis, MO; Timothy J. Davern, Nathan Bass, Smita Rouillard, University 
of California at San Francisco, CA; Evren Atillasoy, Mke Schilsky, Mt Sinai Medical Center, New York, NY; Timothy M. McCashland, University of Nebraska, Omaha, NE; J. Eileen Hay, Mayo Clinic, Rochester, MN; Jeffrey S. Crippin, Baylor University Medical Center, Dallas, TX; A. Obaid Shakil, University of Pittsburgh, PA; Andres T. Blei, Steven Flamm, Northwestern University, Chicago, IL; Kent G. Benner, Hugo R. Rosen, Atif Zaman, Oregon Health Sciences University, Portland, OR; Steven Huy Bui Han, Paul Martin, Risë Stribling, University of California at Los Angeles, CA; Eugene R. Schiff, Maria B. Torres; University of Miami, FL; Robert J. Fontana, University of Michigan, Ann Arbor, MI; Victor Navarro, Yale University, New Haven, CT; Brendan McGuire, University of Alabama at Birmingham, AL; Raymond Chung, Diane Abraczinskas, Jules Dienstag, Massachusetts General Hospital, Boston, MA; Arun Samanta, university of Medicine and Dentistry, Princeton, NJ; Raj Satyanarayana Mayo Clinic, Jacksonville, FLA; Lorenzo Rossaro University of California Davis, Sacremento, CA; Todd Stravitz, Virginia Commonwealth University, Richmond, VA; Santiago Munoz, Albert Einstein Medical Center, Philadelphia, PA; Adrian Reuben, Medical University of South Carolina, Charleston, SC; M Edwyn Harrison, Mayo Clinic, Scottsdale, AZ. This work was supported in part by NIH grant RO1 DK58369.

\section{REFERENCES}

1. Bolzan H, Spatola J, Castelletto R, Curciarello J: Intrahepatic cholestasis induced by amoxicillin alone. Gastroenterol Hepatol 23:237-239, 2000

2. Rodriguez LA, Stricker BH, Zimmerman HJ: Risk of acute liver injury associated with the combination of amoxicillin and clavulanic acid. Arch Intern Med 156:1327-1332, 1996

3. Larrey D, Vial T, Micaleff A, Babany G, Morichau-Beauchant M, Michel H, Benhamou JP: Hepatitis associated with amoxicillinclavulanic acid combination report of 15 cases. Gut 33:368-371, 1992

4. Brown SJ, Desmond PV: Hepatotoxicity of antimicrobial agents. Semin Liver Dis 22:157-167, 2002

5. Hautekeete ML, Brenard R, Horsmans Y, Henrion J, Verbist L, Derue G, Druez P, Omar M, Kockx M, Hubens H: Liver injury related to amoxicillin-clavulanic acid: interlobular bileduct lesion and extrahepatic manifestations. J Hepatol 22:71-77, 1995

6. Higgins PR, Fontana RJ: Liver Transplantation in Acute Liver Failure. Panminerva Med 45:85-94, 2003

7. Ostapowicz G, Fontana RJ, Schiodt FV, Larson A, Davern TJ, Han SH, McCashland TM, Shakil AO, Hay JE, Hynan L, Crippin JS, Blei AT, Samuel G, Reisch J, Lee WM, U.S. Acute Liver Failure Study Group: Results of a prospective study of Acute Liver Failure at 17 Tertiary Care Centers in the United States. Ann Intern Med 137:947-954, 2002

8. Criteria of drug induced liver disorders. Report of an international consensus meeting. J Hepatol 11:272-276, 1990

9. Maria VAJ, Victorino RMM: Development and validation of a clinical scale for the diagnosis of drug induced hepatitis. Hepatology 26:664-669, 1997
10. Danan G, Benichou C: Causality assessment of adverse reactions to drugs-I. A novel method based on the conclusions of international consensus meetings: Application to drug-induced liver injuries. J Clin Epidemiol 46:1331-1336, 1993

11. Benichou, C Danan G. Flahault A: Causality assessment of adverse reaction to drug-II. An original model for validation of drug causality assessment methods: case reports with positive rechallenge. J Clin Epidemiol 46:1323-1330, 1993

12. Aithal GP, Rawlins MD, Day CP: Clinical Diagnostic scale: a useful tool in the evaluation of suspected hepatotoxic adverse drug reactions. J Hepatol 33:949-952, 2000

13. Lucena M, Camargo R, Andrade RJ, Perez-Sanchez CJ, Sanchez De La Cuesta F: Comparison of two clinical scales for causality assessment in hepatotoxicity. Hepatology 33:123-130, 2001

14. Drugtopics.com website: Top 200 drugs in 2002. Accessed October 13, 2003

15. Gresser U: Amoxicillin-clavulinic acid therapy may be associated with severe side effects: Review of the literature. Eur J Med Res 6:139-149, 2001

16. Limauro DL, Chan-Tompkins NH, Carter RW, Brodmerkel GJ, Jr, Agrawal RM: Amoxicillin/clavulanate associated hepatic failure with progression to Stevens-Johnson syndrome. Ann Pharmacother 33:560-564, 1999

17. Ferrando-Vela J, Sanz-Moncasi P, Sevilla-Redondo G, Figueras Villalba P, Martin Algora I: Hepatic failure secondary to hepatitis due to amoxicillin-clavulanic acid. Treatment with corticoids. Ann Med Intern 19(10):551-552, 2002

18. Ersoz G, Karasu Z, Yildiz C, Akarca US, Yuce G, Batur Y: Severe toxic hepatitis associated with amoxicillin and clavulanic acid. J Clin Pharm Ther 26:225-229, 2001

19. Hebbard GS, Smith KG, Gibson PR, Bhathal PS: Augmentin induced jaundice with a fatal outcome. Med J Aust 156:285-228, 1992

20. O’Donohue J. Oien KA, Donaldson P, Underhill J, Clare M, MacSween RN, Mills PR: Co-amoxiclav jaundice: clinical and histological features and HLA class II associations. Gut 47:717-720, 2000

21. Australian Adverse Drug Reactions Bulletin 15:6-7, 1996

22. Shakil AO, Kramer d, Mazariegos GV, Fung JJ, Rakela J: Acute liver failure: clinical features, outcome analysis, and applicability of prognostic criteria. Liver Trans 6:163-169, 2000

23. Larrey D: Epidemiology and individual susceptibility to adverse drug reactions affecting the liver. Semin Liver Dis 22:145-155, 2002

24. Zimmerman HJ: Vulnerability of the liver to toxic injury. In Hepatotoxicity: The adverse effects of drugs and other chemicals on the liver. 2nd ed. Philadelphia, Lippincott, Williams, and Wilkins 1999

25. Van der Auwerea P, Legrand JC. Ticarcillin clavulanic acid therapy in severe infections. Drugs Exp Clin Res Suppl 11:805-813, 1985

26. Reed MD. The clinical pharmacology of amoxicillin and clavulanic acid. Pediatr Infect Dis J 17:957-962, 1989

27. Dinis PB, Monteiro MC, Martins ML, Silva N, Gomes A. Sinus tissue pharmacokinetics after oral administration of amoxicillin/clavulanate acid. Laryngoscope 110:1050-1056, 2000

28. Hautekeete ML, Horsmans Y, Van Waeyenberge C, Demanet C, Henrion J, Verbist L, Brenard R, Sempoux C, Michielsen PP, Yap PS, Rahier J, Geubel AP. HLA association of amoxicillin-clavulanate induced hepatitis. Gastroenterology 117:1181-1186, 1999 